\title{
Research Article \\ Facile Synthesis of Nanoporous Amorphous Silica on Silicon Substrate
}

\author{
Luo Hui $\mathbb{D}^{1}{ }^{1}$ Huang Yongquan, ${ }^{2}$ Wu Lili, ${ }^{3,4}$ Ge Yanming, ${ }^{5}$ Huang Kaichen, ${ }^{1,6}$ Lin Xi, \\ Tang Kexin, ${ }^{1}$ Lv Xiufang, ${ }^{1}$ and Yu Hailing $\mathbb{D}^{1}$ \\ ${ }^{1}$ Guangdong Provincial Key Laboratory of Biomedical Imaging and Guangdong Provincial Engineering Research Center of \\ Molecular Imaging, The Fifth Affiliated Hospital, Sun Yat-sen University, Zhuhai, Guangdong Province 519000, China \\ ${ }^{2}$ Department of Ultrasound, The Fifth Affiliated Hospital, Sun Yat-sen University, Zhuhai, Guangdong Province 519000, China \\ ${ }^{3}$ Key Laboratory for Photonic and Electronic Bandgap Materials, Ministry of Education, School of Physics and Electronic Engineering, \\ Harbin Normal University, Harbin, Heilongjiang Province 150025, China \\ ${ }^{4}$ Center for Engineering Training and Basic Experimentation, Heilongjiang University of Science and Technology, Harbin, \\ Heilongjiang Province 150022, China \\ ${ }^{5}$ Department of Pharmacy, The Fifth Affiliated Hospital, Sun Yat-sen University, Zhuhai, Guangdong Province 519000, China \\ ${ }^{6}$ Institute of Antibody Engineering, School of Laboratory Medicine and Biotechnology, Southern Medical University, Guangzhou, \\ Guangdong Province 510000, China
}

Correspondence should be addressed to Yu Hailing; seal101010@163.com

Received 29 May 2020; Accepted 18 June 2020; Published 14 July 2020

Guest Editor: Zhenyi Zhang

Copyright (C) 2020 Luo Hui et al. This is an open access article distributed under the Creative Commons Attribution License, which permits unrestricted use, distribution, and reproduction in any medium, provided the original work is properly cited.

\begin{abstract}
Large-scale nanoporous amorphous silica nanostructure is fabricated via a simply etched approach and effective thermal evaporation process. The nanoporous amorphous silica was synthesized by a general and scalable process via etching by metal particles on the silica sheets. In this study, we elucidated how a nanoporous structure was performed and the addition of indium is the key factor that determined the formation of the nanoporous structures. The morphology and the sizes of the porous structure could be tunable by the sizes and the shape of the metal. We discovered a promising optical property in the assynthesized nanostructures, which have a photoluminescence in an intensive ultraviolet emission as well as a broad visible emission at room temperature.
\end{abstract}

\section{Introduction}

Silica materials exhibit unique physical, mechanical, and chemical properties that make them attractive, for example, for heterogeneous catalysis [1-3], drug delivery [4], and composites [5]. Amorphous silica networks [6, 7] have a high surface area, low density [6], good dispersity, and unique morphology which could be widely used in the application of gas and liquid separation [8] and catalysis carrier [9]. The common method to get amorphous silica network is CVD and sol-gel process [10-13]. However, it is difficult to get pure inorganic oxides in the amorphous silica networks acquired by the sol-gel process, which includes the hydrolytic polycondensation of appropriate liquid precursors [14-16]. The obtained amorphous silica always contains hydroxyl $(-\mathrm{OH})$ and ethoxy $\left(-\mathrm{OCH}_{2} \mathrm{CH}_{3}\right)$ groups, and the structure is chemically instable because of the condensation of the remaining $\mathrm{OH}$ group. The pore size of an amorphous silica film by the sol-gel process is determined by the polymeric and colloidal routes, which is still a challenge to obtain pores of small size. And the sol-gel process uses high-cost precursors such as tetraethoxysilane (TEOS) and the drying of the gels, which is hard to get large-scale production [13]. A simple method to produce pure porous silica is essential for a number of applications $[2,17,18]$.

The pore engineering on graphene sheets through carbothermal reaction provides a universal and economical 
method for the synthesis of porous nanostructures [19]. Herein, we demonstrate a simple and scalable approach to the fabrication of large-scale, nanoporous amorphous silica structure. The nanoporous amorphous silica is formed via metal droplet etching that resulted in the vacancy formation. And the as-synthesized nanostructures have a strong ultraviolet emission in photoluminescence spectrum at room temperature.

\section{Experimental}

First, the nickel films were deposited on the silicon substrate by a high-vacuum magnetron sputtering systems. Before being deposited, silicon substrate is cleaned by acetone in an ultrasonic bath for $10 \mathrm{~min}$, rinsed with isopropanol, and dried with argon. The deposition time of the nickel films is $20 \mathrm{~min}$, and the constant power density of the nickel target is $5.3 \mathrm{~W} / \mathrm{cm}^{2}$. The gas pressure was maintained at $1.0 \mathrm{~Pa}$ during deposition, and Ar flow rate was adjusted to 100 SCCM. The silicon substrate covered with nickel film and indium powder was annealed at $1000^{\circ} \mathrm{C}$ for 3 hours. The pressure during the heat treatment was $0.001 \mathrm{~Pa}$, and we naturally cooled the sample to room temperature in the furnace.

The morphology of the samples was observed by scanning electron microscopy (SEM) and transmission electron microscopy (TEM). The samples were characterized using a TEM (Tecnai G2 F30, operated at $300 \mathrm{kV}$ ) and SEM (Helios Nanolab 660i, operated at $20 \mathrm{kV}$ ). The structure of samples was analyzed using grazing incidence X-ray diffractometer (GIXRD, PANalytical X'Pert ProCu K $\alpha$ ). The sample for TEM was dispersed using ultrasonic dispersion. The Raman spectrum of the samples was measured using a micro-Raman spectrometer (LabRAM HR800) using a $\mathrm{He}-\mathrm{Cd}$ laser with an excitation wavelength of $325 \mathrm{~nm}$ at room temperature.

\section{Results and Discussion}

3.1. Structure and Morphology Characterization. Figures 1(a) and 1(b) are the typical SEM images of the obtained silica film. Large-scale porous nanostructures with foam-like morphology were stacked on the silicon substrate. The nanoporous film with thickness from several tens of nanometers up to $1 \mu \mathrm{m}$ and size can be scaled up by enlarging the silicon substrate. The pores in the network are not uniform, and the sizes of the pores vary from several nanometers to hundreds of nanometers. There is a direct relationship between the sizes of the pores and the diameters of the particles inside the holes. The statistical analysis of the size distributions for the particles and the corresponding holes indicates that the diameters of the particles inside and the sizes of the corresponding pores are linear. The pores always have similar shape like the particles inside. And the area of porous nanostructure decreased with the amount of particles increasing. The histogram shows that the majority of the particles have a diameter in range of $10 \mathrm{~nm}-400 \mathrm{~nm}$. However, we cannot observe some particles in the smaller pores because the small particles are easy to be removed or covered by the silica films. There are two or more particles in the large holes. The results reveals that the particles are the determining factor in the formation of the nanoporous structure and it is possible to tune pore sizes and morphology of the nanostructure by tailoring the sizes and shape of particles. It is shown that the diameters of most droplets are within a range of $10-300 \mathrm{~nm}$ in the SEM image and the element ratio of the particle is $\mathrm{Ni}: \mathrm{In}=2: 3$ characterized by EDS. The results show that the formed $\mathrm{Ni}_{2} \mathrm{In}_{3}$ alloy particles have the diameters of tens of nanometers and a crucial effect on the formation of porous structures. The formation mechanism of the porous nanostructure will be discussed in the following section.

Figures 2(a) and 2(b) shows the TEM images of the nanoporous film. It is obvious that the nanostructure has networklike morphology. The porous nanostructure is composed of multilayer films with nanoporous structure, and the edges of the films reel up to a nanotube marked by an arrow in Figure 2(a). The particles which are shown from the SEM image are not observed in TEM images. Because the samples for TEM have been dispersed by ultrasonic dispersion, maybe the particles are gone during this process. It revealed that it is easy to remove the particles for pure porous amorphous silica by ultrasonic dispersion. No crystal structure was found in the high-resolution transmission electron microscopy mode, which suggests that the films are amorphous. It is demonstrated that the porous nanostructure includes silicon, oxygen, and copper that comes from the grid by EDS. Figure 3(a) is the XRD pattern of the porous nanostructures. The XRD pattern shows that the sample is amorphous in a $15^{\circ}-35^{\circ}$ region. The broad peak in the range of $15^{\circ}$ to $30^{\circ}$ in the XRD pattern always confirms that the silica is amorphous [20]. The small diffraction peak corresponds to the silicon substrate, and the other peaks could not be distinguished for the XRD pattern because of the broad amorphous peak. The SAED image of the porous nanostructures is shown in Figure 2(c). There is not any trace of crystal structure, and the highly diffusive ring pattern indicates that the structure is of a completely amorphous state. The result from the SEAD was in accord with TEM and EDS analysis of the porous compositions that indicated that the nanostructure contains only silicon and oxygen elements.

The photoluminescence (PL) spectrum of porous amorphous silica films is showed in Figure 3(b), excited by $325 \mathrm{~nm}$ wavelength. The porous silica has a broadband spectrum from ultraviolet to visible light. There are a sharp peak located at $358 \mathrm{~nm}(3.46 \mathrm{eV})$ and a broad peak in the $450 \mathrm{~nm}$ to $650 \mathrm{~nm}$ band. It is proposed that two folded coordination silicon lone pair centers $(\mathrm{O}-\mathrm{Si}-\mathrm{O})$ and neutral oxygen vacancies $(\equiv \mathrm{Si}-\mathrm{Si} \equiv)$ are the sources of amorphous silica [21]. It has been indicated that the silica exhibits visible PL emission under UV excitation, resulting from the nonbridging oxygen hole centers and hydrogen-related species on the surface of silica [22-24]. These defects are clearly caused by the amorphous structure of the samples. These structure defects can be radiative recombination centers, and ultraviolet light emission in annealed $\mathrm{SiO}_{2}$ is already observed [21]. So the intensive ultraviolet light 


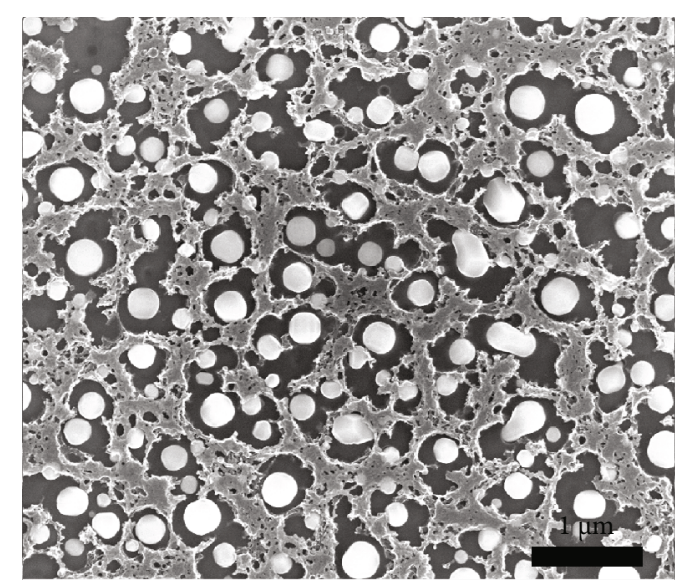

(a)

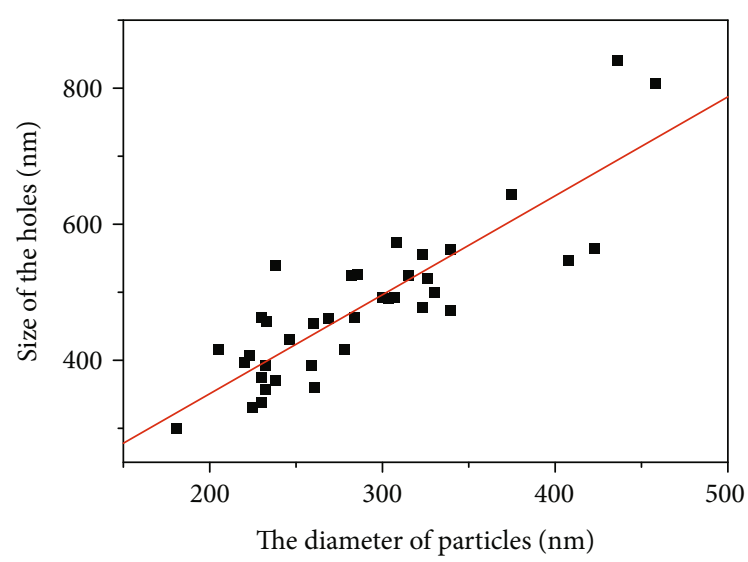

(c)

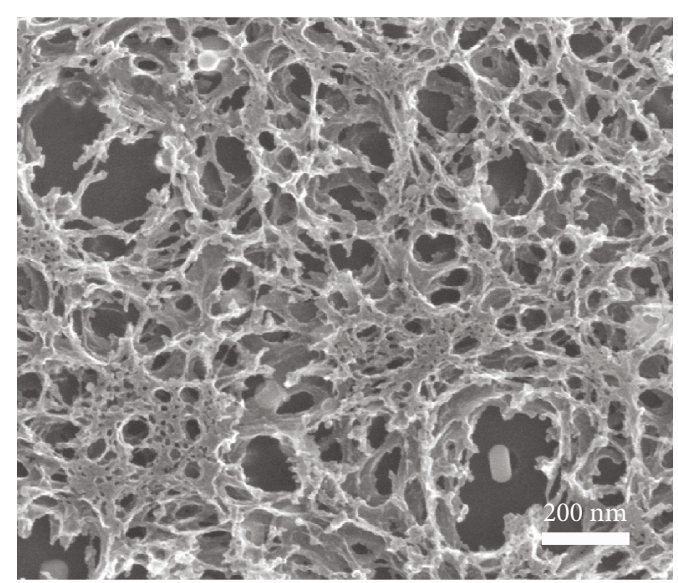

(b)

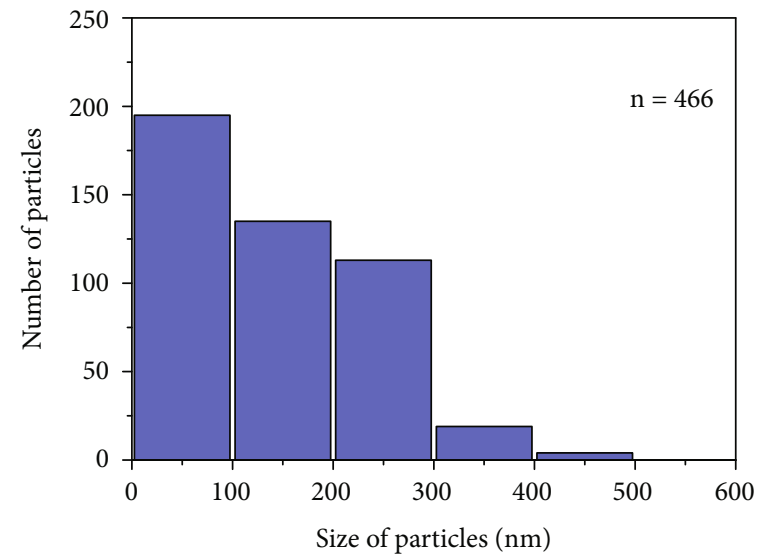

(d)

FIgURE 1: (a) SEM images of nanoporous silica. (b) Magnified SEM images of nanoporous silica. (c) The statistical analyses of the size distributions for the particles and the corresponding holes. (d) The histogram of the particles for SEM image.

emission from the porous amorphous silica is attributable to $\mathrm{Si}-\mathrm{O}$ defects [25]. The ultraviolet emission silica films may be potentially used as promising UV laser emitters.

3.2. Growth Mechanism. In this study, most of the nanoporous nanostructures were presentence of alloy particles in the pores as shown in Figure 1, indicating that nanoporous silica is etched by the metal nanoparticles; nanoscaled pores are generated on the silica film. To better explain the growth mechanism of the nanoporous silica, the possible mechanism of the porous amorphous nanostructure is proposed in Figure 4 . The porous silica film is caused by Ni-In metal particles interspersed on its surface. In was liquefied at $157^{\circ} \mathrm{C}$ that is absorbed by nickel, and $\mathrm{Ni}_{2} \mathrm{In}_{3}$ is formed above $448^{\circ} \mathrm{C}$. The melting temperature of $\mathrm{Ni}_{2} \mathrm{In}_{3}$ is $448^{\circ} \mathrm{C}$; therefore, it is in a liquid form in the temperature of $1000^{\circ} \mathrm{C}$. The heating process is long enough to reach saturation, and indium power is easy to evaporate during the thermal process. The oxygen atoms remaining in the low vacuum can form $\mathrm{SiO}_{2}$ with silicon atoms at high temperature. Simultaneously, the indium atoms from the indium powder reacted with nickel to form $\mathrm{Ni}$-In spherical island that resulted from the effect of the surface tension. The Si-O atoms are difficult to crystal- lize at low temperature, leading to the formation of the amorphous silica rather than crystalline silica. Nanoscaled pores could be observed under nanoparticles, validating the pore generation through the etching by the metal nanoparticles. The $\mathrm{Ni}_{2} \mathrm{In}_{3}$ alloy and silica are to a certain extent immiscible that EDS analysis indicated that the particles are only including $\mathrm{Ni}$ and $\mathrm{In}$ elements. The $\mathrm{Ni}_{2} \mathrm{In}_{3}$ alloy can be removed easily and leave a stable and porous phase left. Indium is critical for the formation of porous silica, and the obtained nanostructure could be nanowire with $\mathrm{Ni}-\mathrm{Si}$ alloy at the top of the wire, if no catalyst indium was added during thermal process in our previous literatures [26, 27]. The formation of the porous structure on the silica film resulted from an etched approach by using the alloy particles as nanoscale etcher. The shape and size of the $\mathrm{Ni}_{2} \mathrm{In}_{3}$ liquid alloy determine the porous structure of the silica film. Therefore, the sizes of pores are affected by the size of an original diameter of the Ni-In droplet.

\section{Conclusion}

In this study, we demonstrated a feasible method to produce nanoporous amorphous silica by using the alloy particles as 


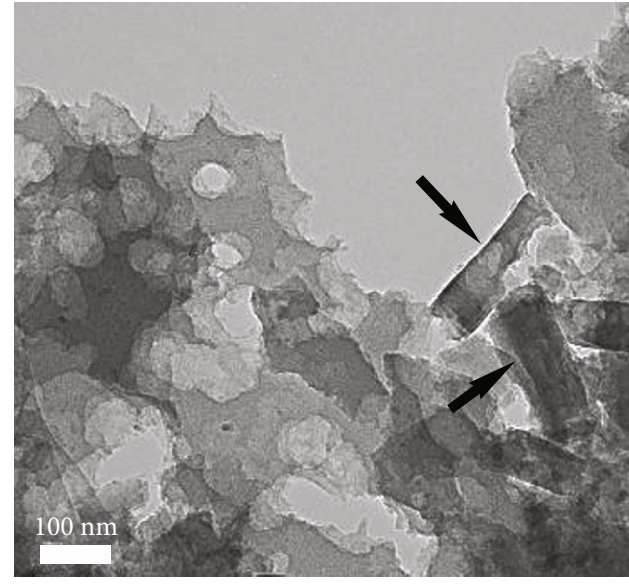

(a)

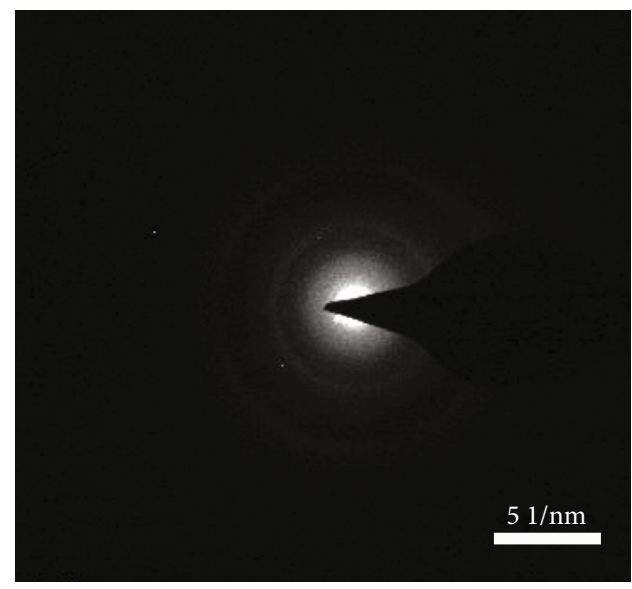

(c)

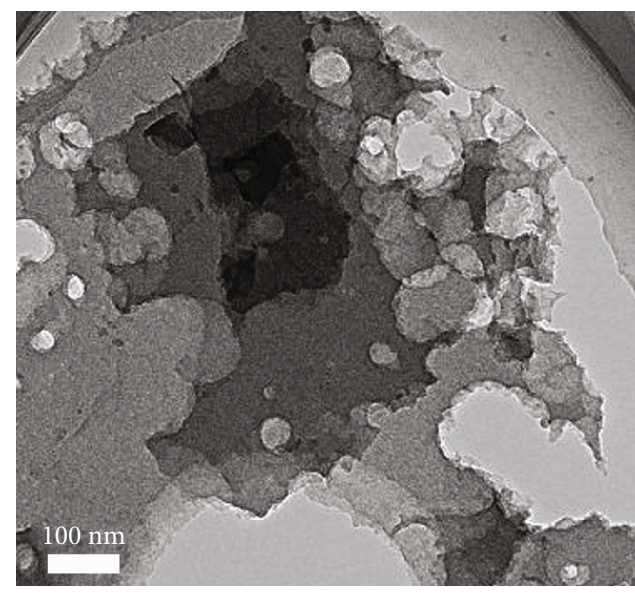

(b)

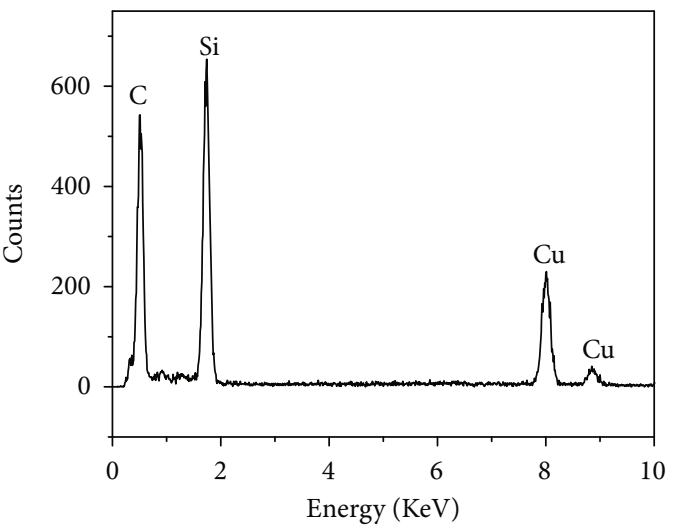

(d)

FIgURE 2: (a, b) TEM images of nanoporous silica. (c) The SAED of nanoporous silica. (d) The EDS analysis of the nanoporous silica.

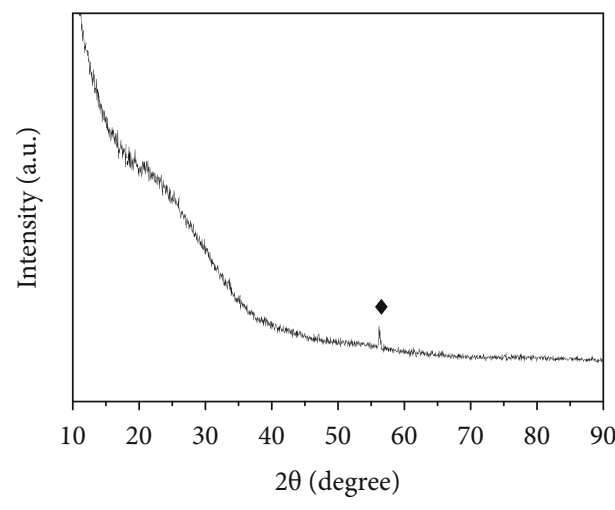

- Silicon substrate

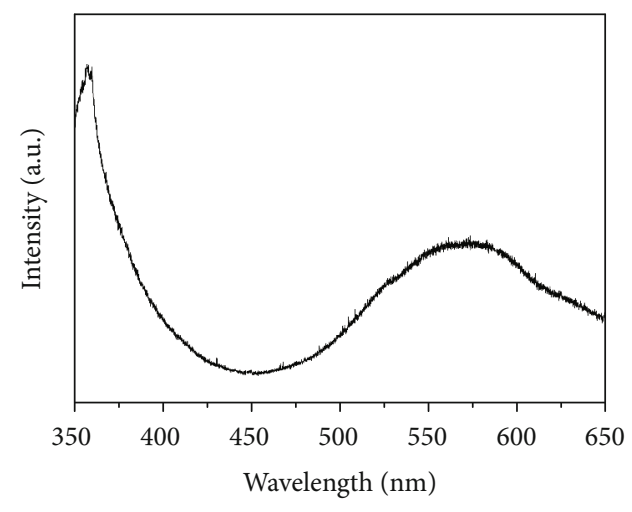

(b)

FIGURE 3: (a) XRD pattern of nanoporous silica networks. (b) The PL spectrum of obtained simples.

nanoscale etcher. The pore sizes varied from hundreds to several tens of nanometers, and the thickness of nanostructures is up to micrometers. The alloy droplet is the key factor that determined the formation of the nanoporous silica, and the sizes of the pores are linear with those of the alloy droplet insides. The amorphous silica has a strong peak in the 

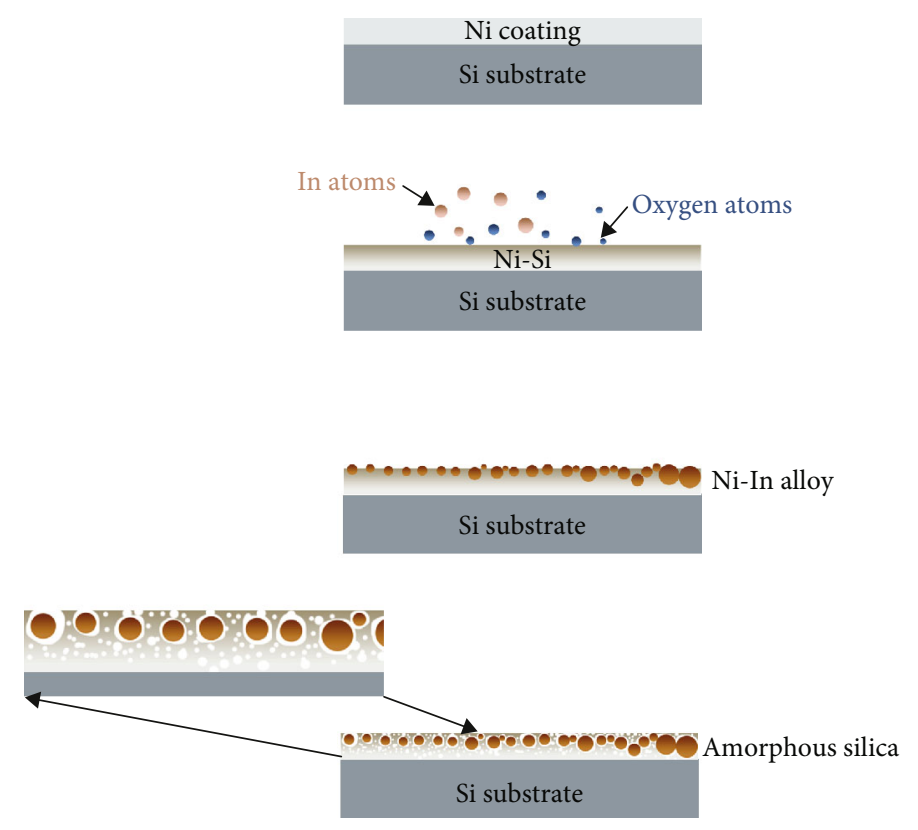

FIGURE 4: Schematic of the nanoporous amorphous silica.

ultraviolet band and a weak visible peak that may be caused by the defect centers in amorphous silica. The porous amorphous silica films would be a good UV emitter in applications such as light-emitting diodes or displays.

\section{Data Availability}

All the data used to support the findings of this study are available from the corresponding author upon request.

\section{Conflicts of Interest}

The authors declare that they have no conflicts of interest.

\section{Authors' Contributions}

Luo Hui and Huang Yongquan contributed equally to this work.

\section{Acknowledgments}

This paper is supported by the Fundamental Research Funds for the Central Universities (19ykpy42); the Guangdong Basic and Applied Basic Research Foundation (2020A1515011001); the Natural Science Foundation of Heilongjiang Province, China (no. QC2017003); the Scientific Research Foundation of Heilongjiang Province for Returned Chinese Scholars (Wu Li Li); Scientific research project of Traditional Chinese Medicine Bureau of Guangdong Province (20201071); and the Guangdong Basic and Applied Basic Research Fund Regional Joint Fund-Youth Fund Project (2019A1515110457).

\section{References}

[1] S. Inagaki, H. Imai, S. Tsujiuchi, H. Yakushiji, T. Yokoi, and T. Tatsumi, "Enhancement of catalytic properties of interlayer-expanded zeolite Al-MWW via the control of interlayer silylation conditions," Microporous and Mesoporous Materials, vol. 142, no. 1, pp. 354-362, 2011.

[2] Y. Wang, J. Liu, Y. Wang, and M. Zhang, " $\mathrm{CO}_{2}$ photoreduction to $\mathrm{CO} / \mathrm{CH}_{4}$ over $\mathrm{Bi}_{2} \mathrm{~W}_{0.5} \mathrm{Mo}_{0.5} \mathrm{O}_{6}$ solid solution nanotubes under visible light," RSC Advances, vol. 10, no. 15, pp. 88218824, 2020.

[3] D. Ballivet-Tkatchenko, F. Bernard, F. Demoisson, L. Plasseraud, and S. R. Sanapureddy, "Tin-based mesoporous silica for the conversion of $\mathrm{CO}_{2}$ into dimethyl carbonate," ChemSusChem, vol. 4, no. 9, pp. 1316-1322, 2011.

[4] J. Muzik, D. Lizonova, A. Zadrazil, and F. Stepanek, "Drug amorphisation by fluid bed hot-melt impregnation of mesoporous silica carriers," Chemical Engineering Journal, vol. 392, article 123754, 2020.

[5] J. W. Park, H. Y. Park, J. Kang, S. Kim, and Y. Jung, "Carbon nanotube-based sulfur cathode with a mesoporous carbonsilica composite for long cycle life Li-S batteries," Journal of Nanoscience and Nanotechnology, vol. 20, no. 8, pp. 49494954, 2020.

[6] M. Perez-Garnes, M. Gutierrez-Salmeron, V. Morales et al., "Engineering hollow mesoporous silica nanoparticles to increase cytotoxicity," Materials Science and Engineering: C, vol. 112, pp. 110935-110935, 2020.

[7] A. Szczurek, M. Paszkowski, D. Lewandowski, J. Gasiorek, J. Kaleta, and J. Krzak, "Organically functionalized sol-gel silica network growth," Ceramics International, vol. 46, no. 9, pp. 13198-13204, 2020.

[8] Z. Y. Yeo, T. L. Chew, P. W. Zhu, A. R. Mohamed, and S.-P. Chai, "Synthesis and performance of microporous inorganic membranes for $\mathrm{CO}_{2}$ separation: a review," Journal of Porous Materials, vol. 20, no. 6, pp. 1457-1475, 2013. 
[9] D. Esquivel, J. Amaro-Gahete, N. Caballero-Casero et al., "Tailoring bifunctional periodic mesoporous organosilicas for cooperative catalysis," ACS Applied Nano Materials, vol. 3, no. 3, pp. 2373-2382, 2020.

[10] L. Huang and J. Kieffer, "Amorphous-amorphous transitions in silica glass. II. Irreversible transitions and densification limit," Physical Review B, vol. 69, no. 22, article 224204, 2004.

[11] C. J. Johnston, K. E. Driscoll, J. N. Finkelstein et al., "Pulmonary chemokine and mutagenic responses in rats after subchronic inhalation of amorphous and crystalline silica," Toxicological Sciences, vol. 56, no. 2, pp. 405-413, 2000.

[12] A. Wootton, B. Thomas, and P. Harrowell, "Radiationinduced densification in amorphous silica: a computer simulation study," Journal of Chemical Physics, vol. 115, no. 7, pp. 3336-3341, 2001.

[13] R. Ciriminna, A. Fidalgo, V. Pandarus, F. Beland, L. M. Ilharco, and M. Pagliaro, "The sol-gel route to advanced silica-based materials and recent applications," Chemical Reviews, vol. 113, no. 8, pp. 6592-6620, 2013.

[14] J. Chen, C. Sun, Z. Huang, F. Qin, H. Xu, and W. Shen, "Fabrication of functionalized porous silica nanocapsules with a hollow structure for high performance of toluene adsorption-desorption," ACS Omega, vol. 5, no. 11, pp. 5805-5814, 2020.

[15] P. Zhao, G. Zhang, and L. Hao, "A novel blended amine functionalized porous silica adsorbent for carbon dioxide capture," Adsorption, vol. 26, no. 5, pp. 749-764, 2020.

[16] D. Zhao, J. Feng, Q. Huo et al., "Triblock copolymer syntheses of mesoporous silica with periodic 50 to 300 angstrom pores," Science, vol. 279, no. 5350, pp. 548-552, 1998.

[17] S. Chen, M. Zhang, X. Ma, L. Li, X. Zhou, and Z. Zhang, "Asymmetric supercapacitors by integrating high content $\mathrm{Na}^{+} / \mathrm{K}^{+}$-inserted $\mathrm{MnO}_{2}$ nanosheets and layered $\mathrm{Ti}_{3} \mathrm{C}_{2} \mathrm{~T}_{\mathrm{x}}$ paper," Electrochimica Acta, vol. 332, article 135497, 2020.

[18] J. Huang, T. Liu, R. Wang et al., "Facile loading of cobalt oxide on bismuth vanadate: proved construction of $\mathrm{p}-\mathrm{n}$ junction for efficient photoelectrochemical water oxidation," Journal of Colloid and Interface Science, vol. 570, pp. 89-98, 2020.

[19] D. Zhou, Y. Cui, P. W. Xiao, M. Y. Jiang, and B. H. Han, “A general and scalable synthesis approach to porous graphene," Nature Communications, vol. 5, no. 1, article 4716, 2014.

[20] K. Liu, Q. Feng, Y. Yang, G. Zhang, L. Ou, and Y. Lu, "Preparation and characterization of amorphous silica nanowires from natural chrysotile," Journal of Non-Crystalline Solids, vol. 353, no. 16-17, pp. 1534-1539, 2007.

[21] H. Nishikawa, T. Shiroyama, R. Nakamura, Y. Ohki, K. Nagasawa, and Y. Hama, "Photoluminescence from defect centers in high-purity silica glasses observed under 7.9-eV excitation," Physical Review B, vol. 45, no. 2, pp. 586-591, 1992.

[22] Y. D. Glinka, S.-H. Lin, and Y.-T. Chen, "The photoluminescence from hydrogen-related species in composites of $\mathrm{SiO}_{2}$ nanoparticles," Applied Physics Letters, vol. 75, no. 6, pp. 778-780, 1999.

[23] Y. D. Glinka, S. H. Lin, L. P. Hwang, and Y. T. Chen, "Photoluminescence from mesoporous silica: similarity of properties to porous silicon," Applied Physics Letters, vol. 77, no. 24, pp. 3968-3970, 2000.

[24] Y. D. Glinka, S. H. Lin, and Y. T. Chen, "Time-resolved photoluminescence study of silica nanoparticles as compared to bulk
type-III fused silica," Physical Review B, vol. 66, no. 3, article 035404, 2002.

[25] Z. Xiao, L. Zhang, G. Meng, X. Tian, H. Zeng, and M. Fang, "High-density, aligned $\mathrm{SiO}_{2}$ nanowire arrays: microscopic imaging of the unique growth style and their ultraviolet light emission properties," The Journal of Physical Chemistry B, vol. 110, no. 32, pp. 15724-15728, 2006.

[26] H. Yu, X. Ren, Y. Huang et al., "The controllable growth of superhydrophobic $\mathrm{SiC}$ nanowires by tailoring the cooling rate," CrystEngComm, vol. 20, no. 47, pp. 7706-7712, 2018.

[27] H. Yu, J. Zhu, L. Yang et al., "Superhydrophobic carbon nanotube/silicon carbide nanowire nanocomposites," Materials \& Design, vol. 87, pp. 198-204, 2015. 